PROCEEDINGS OF THE

AMERICAN MATHEMATICAL SOCIETY

Volume 135, Number 1, January 2007, Pages 51-57

S 0002-9939(06)08532-7

Article electronically published on July 28, 2006

\title{
A LINEAR COUNTEREXAMPLE TO THE FOURTEENTH PROBLEM OF HILBERT IN DIMENSION ELEVEN
}

\author{
GENE FREUDENBURG
}

(Communicated by Bernd Ulrich)

\begin{abstract}
A family of $\mathbb{G}_{a}$-actions on affine space $\mathbb{A}^{m}$ is constructed, each having a non-finitely generated ring of invariants $(m \geq 6)$. Because these actions are of small degree, they induce linear actions of unipotent groups $\mathbb{G}_{a}^{n} \rtimes \mathbb{G}_{a}$ on $\mathbb{A}^{2 n+3}$ for $n \geq 4$, and these invariant rings are also non-finitely generated. The smallest such action presented here is for the group $\mathbb{G}_{a}^{4} \rtimes \mathbb{G}_{a}$ acting linearly on $\mathbb{A}^{11}$.
\end{abstract}

\section{INTRODUCTION}

More than a century after it was first posed, we are still sounding the depths of Hilbert's Fourteenth Problem. In its geometric form, this asks whether the invariant ring of a $G$-variety $V$ is finitely generated over its base field, with particular interest in the case $V$ is a $G$-module. Nagata's counterexamples from 1958 use the commutative vector group $\mathbb{G}_{a}^{13}$ acting on the affine space $\mathbb{A}^{32}$ by linear transformations [14. Since then, other counterexamples have been found that show that both the dimension of the group $G$ which acts, as well as the dimension of the affine space $\mathbb{A}^{n}$ on which it acts can be reduced. However, there remain several key interrelated questions regarding minimality of dimension in any counterexample to the Fourteenth Problem.

These questions are framed by the following three finiteness theorems, where $k$ denotes the base field. When $G$ is an algebraic $k$-group acting algebraically on an algebraic $k$-variety $X$, then $k[X]$ is the coordinate ring of $X$, and $k[X]^{G}$ its ring of invariants. The additive group of the field $k$ is denoted by $\mathbb{G}_{a}$, and its multiplicative group of units by $\mathbb{G}_{m}$.

Finiteness Theorem. If $G$ is any finite or reductive group acting algebraically on a variety $X$, then $k[X]^{G}$ is finitely generated over $k$ (see [3]).

Zariski Finiteness Theorem. If $k\left(x_{1}, \ldots, x_{n}\right)$ is the rational function field in $n$ variables over $k$, and $K$ is a subfield containing $k$ such that $\operatorname{tr} \cdot \operatorname{deg} \cdot{ }_{k} K \leq 2$, then $K \cap k\left[x_{1}, \ldots, x_{n}\right]$ is finitely generated over $k[18$.

Received by the editors August 10, 2005.

2000 Mathematics Subject Classification. Primary 13A50, 14R20.

Key words and phrases. Hilbert's Fourteenth Problem, invariant theory, locally nilpotent derivations.

(C)2006 American Mathematical Society Reverts to public domain 28 years from publication 
Maurer-Weitzenböck Theorem. If $k$ is of characteristic zero, and $G$ is a onedimensional group acting on $V=\mathbb{A}^{n}$ by linear transformations, then $k[V]^{G}$ is finitely generated over $k$ (see [5]).

Thus, when the characteristic of $k$ is 0 , a linear action of $\mathbb{G}_{a}$ on $\mathbb{A}^{n}$ has a finitely generated ring of invariants. On the other hand, working over the complex field, Mukai has constructed linear actions of the three-dimensional vector group $\mathbb{G}_{a}^{3}$ on $\mathbb{A}^{18}$, which have non-finitely generated rings of invariants [13].

Question 1. Is the invariant ring of a linear $\mathbb{G}_{a}^{2}$-action on $\mathbb{A}^{n}$ always finitely generated? Equivalently (over a field of characteristic 0): Is the common kernel of two commuting nilpotent matrices always of finite type?

Remarkably, in the case of positive characteristic, the situation for linear $\mathbb{G}_{a^{-}}$ actions is still completely open.

Question 2. Over a field of positive characteristic, is the invariant ring of a linear $\mathbb{G}_{a}$-action on $\mathbb{A}^{n}$ always finitely generated?

The first (non-linear) $\mathbb{G}_{a}$-action on affine space with non-finitely generated invariant ring was constructed by P. Roberts [15], specifically using $\mathbb{A}^{7}$ over a field of characteristic 0 . A similar counterexample was later given by the author and Daigle using a $\mathbb{G}_{a}$-action on $\mathbb{A}^{5}[2$. Note that, due to the Zariski Finiteness Theorem, any $G$-action on $\mathbb{A}^{3}$ will have $k\left[\mathbb{A}^{3}\right]^{G}$ finitely generated. This leaves:

Question 3. Is the invariant ring of a $\mathbb{G}_{a}$-action on $\mathbb{A}^{4}$ always finitely generated?

A related question is:

Question 4. Suppose that a $G$-action on $\mathbb{A}^{n}$ has non-finitely generated invariant ring $k\left[\mathbb{A}^{n}\right]^{G}$. Can tr.deg. $k\left[\mathbb{A}^{n}\right]^{G}$ be equal to 3 ?

Several known examples, including one of Nagata's, have invariant rings of transcendence degree 4 over $k$. On the other hand, the Zariski Finiteness Theorem implies that no counterexample can have invariant ring of transcendence degree 2 over $k$. Recently, Kuroda constructed counterexamples to the more general version of the Fourteenth Problem that use a subfield of a rational function field having transcendence degree 3 over $k$ (see [9, 11]). But for invariant rings of group actions, this remains an open question.

Following Nagata's counterexample for $\mathbb{A}^{32}$, a series of linear counterexamples to the Fourteenth Problem on smaller affine spaces were found: A'Campo-Neuen for $\mathbb{A}^{19}\left([1,1994)\right.$, Steinberg for $\mathbb{A}^{18}\left([16,1997)\right.$, Mukai for $\mathbb{A}^{16}([13,2002)$, and Tanimoto for $\mathbb{A}^{13}($ [17, 2004) 1$]$

Question 5. What is the smallest value of $n$ such that, for some group $G$, there exists a linear algebraic $G$-action on $\mathbb{A}^{n}$ with non-finitely generated ring of invariants?

Note that the Zariski Finiteness Theorem, together with the Maurer-Weitzenböck Theorem, imply that $n \geq 5$ in this situation.

The purpose of this brief article is twofold: To bring greater attention to these fascinating questions, and to provide an example showing that the value of $n$ may be reduced to $n=11$ in the known examples for the last question.

\footnotetext{
${ }^{1}$ In the examples of Nagata and Steinberg, $k$ is any field that is not locally finite; for A'CampoNeuen and Tanimoto, $k$ is any field of characteristic zero; and in Mukai's examples, $k=\mathbb{C}$.
} 
Specifically, the last section of the paper gives a linear action of the unipotent group $\mathbb{G}_{a}^{4} \rtimes \mathbb{G}_{a}$ on $\mathbb{A}^{11}$ having non-finitely generated ring of invariants. This example is based on recent work of Kuroda [10, who constructed a family of non-linear counterexamples generalizing those of Roberts. These are $\mathbb{G}_{a}$-actions on affine spaces that, although not linear, are of a particularly simple form called elementary. In Section 2, we will show that the Kuroda examples induce a family of nonelementary $\mathbb{G}_{a}$-actions in smaller dimension. Finally, these $\mathbb{G}_{a}$-actions are used to define linear actions of larger groups on larger affine spaces, and these linear actions also have invariant rings, which are not finitely generated. The key fact is that the degrees for the intermediate $\mathbb{G}_{a}$-actions are small, meaning that the induced linear actions can be defined on $\mathbb{A}^{n}$ for $n \geq 11$.

The notation $R^{[n]}$ will denote the polynomial ring in $n$ variables over the ring $R$.

I wish to thank Robert Steinberg and Ryuji Tanimoto for their helpful comments about this paper.

\section{The Roberts Derivations}

Fix a field $k$ of characteristic 0 . The reader is reminded that $\mathbb{G}_{a}$-actions on a $k$-variety $X$ correspond to locally nilpotent derivations $D$ on the coordinate ring $k[X]$; see for example [4] or [5]. In particular, if $D$ is a locally nilpotent derivation of $k[X]$, the corresponding $\mathbb{G}_{a}$-action on $X$ is $\exp (t D), t \in \mathbb{G}_{a}$, and the ring of invariants $k[X]^{\mathbb{G}_{a}}$ equals the ring of constants (kernel) for the derivation, denoted $k[X]^{D}$ or $\operatorname{ker}(D)$. When $X=\mathbb{A}^{n}$, a linear $\mathbb{G}_{a}$-action is given by a unipotent matrix of order $n$, which is the exponential of a nilpotent matrix.

The simplest non-linear locally nilpotent derivations of a polynomial ring are the elementary derivations. Specifically, let $B=k\left[x_{1}, \ldots, x_{n}\right]$ be the polynomial ring in $n$ variables over $k$. Then a $k$-derivation $D$ of $B$ is elementary if and only if, for some $i, 1 \leq i \leq n, D$ has the form

$$
D x_{j}=0 \text { for } 1 \leq j \leq i, \quad \text { and } D x_{j} \in k\left[x_{1}, \ldots, x_{i}\right] \text { for } i<j \leq n .
$$

If, in addition, each image $D x_{j}$ is a monomial in $x_{1}, \ldots, x_{i}$, then $D$ is called elementary monomial.

Already among the $\mathbb{G}_{a}$-actions associated to elementary monomial derivations, we find counterexamples to the Fourteenth Problem. The first of these is due to Roberts 15]; subsequent work on this subject is found in [4, 6, 7, 12.

A recent paper of Kuroda 10 also studies this class of derivations. In it the author gives an inequality determined by the defining monomials of an elementary monomial derivation, and proves that when this inequality is satisfied, the ring of constants for $D$ is not finitely generated ([10, Theorem 1.3]).

Of particular interest is the following special case. Let $B=k[\mathbf{x}, \mathbf{y}, z]=k^{[2 n+1]}$, where $\mathbf{x}=\left(x_{1}, \ldots, x_{n}\right)$ and $\mathbf{y}=\left(y_{1}, \ldots, y_{n}\right)$ for $n \geq 1$. Given an integer $t \geq 1$, define the Roberts derivation $D_{(t, n)}$ on $B$ by:

$$
D_{(t, n)}(\mathbf{x})=0 ; D_{(t, n)}\left(y_{i}\right)=x_{i}^{t+1}(1 \leq i \leq n) ; \text { and } D_{(t, n)}(z)=\left(x_{1} \cdots x_{n}\right)^{t} .
$$

Theorem 2.1. If $n \geq 3$ and $t \geq 2$, or if $n \geq 4$ and $t \geq 1$, then the ring of constants of $D_{(t, n)}$ is not finitely generated.

The original examples of Roberts were $D_{(t, 3)}$ for $t \geq 2$. Then Kojima and Miyanishi proved the result for the case $n \geq 3$ and $t \geq 2$ [7]. Finally, Kuroda proved all cases of the theorem, including the case $n \geq 4$ and $t \geq 1$ ([10], Cor. 1.5). 
It is this last case, which is new, that serves as the motivation for this paper: We consider the Roberts derivations $D_{(1, n)}$ for $n \geq 4$.

It should be noted that Kurano showed that the ring of constants for $D_{(1,3)}$ is generated by 9 elements 8 .

\section{Triangular DERIVATIONS INDUCED By Roberts DERIVATIONS}

The basic representation of $\mathbb{G}_{a}$ on $\mathbb{A}^{N}$ is defined by the exponential of the elementary nilpotent matrix

$$
E_{N}=\left(\begin{array}{ccccc}
0 & 1 & 0 & \cdots & 0 \\
0 & 0 & 1 & \cdots & 0 \\
\vdots & \vdots & \ddots & \ddots & \vdots \\
0 & 0 & 0 & \ddots & 1 \\
0 & 0 & 0 & \cdots & 0
\end{array}\right)_{N \times N}
$$

Let $V_{N}$ denote $\mathbb{A}^{N}$ as a $\mathbb{G}_{a}$-module with this action. The purpose of this section is to prove the following.

Theorem 3.1. Given $N \geq 5$, let $k[\mathbf{u}, \mathbf{v}]=k^{[N+2]}$ denote the coordinate ring of $V_{2} \oplus V_{N}$, where $\mathbf{u}=\left(u_{1}, u_{2}\right)$ and $\mathbf{v}=\left(v_{1}, \ldots, v_{N}\right)$, and $E_{2}\left(u_{1}\right)=E_{N}\left(v_{1}\right)=0$. Let $X \subset \mathbb{A}^{N+2}$ denote the coordinate hypersurface defined by the invariant function $\left(v_{1}-u_{1}^{2}\right)$. Then $\mathbb{G}_{a}$ acts on $X$ by restriction, and $k[X]^{\mathbb{G}_{a}}$ is not finitely generated.

Proof. Given $n \geq 4$, let $\delta_{n}$ denote the Roberts derivation $D_{(1, n)}$ on the polynomial ring $k[\mathbf{x}, \mathbf{y}, z]=k^{[2 n+1]}$, where $\mathbf{x}=\left(x_{1}, \ldots, x_{n}\right)$ and $\mathbf{y}=\left(y_{1}, \ldots, y_{n}\right)$. Specifically,

$$
\delta_{n}=\left(\sum_{1 \leq i \leq n} x_{i}^{2} \frac{\partial}{\partial y_{i}}\right)+\left(x_{1} \cdots x_{n}\right) \frac{\partial}{\partial z} .
$$

By Kuroda's result, the ring of constants of $\delta_{n}$ is not finitely generated.

This derivation has many symmetries. First, there is an obvious action of the torus $\mathbb{G}_{m}^{n}$ on $k[\mathbf{x}, \mathbf{y}, z]$ that commutes with $\delta_{n}$, namely, given $\lambda=\left(\lambda_{1}, \ldots, \lambda_{n}\right) \in \mathbb{G}_{m}^{n}$,

$\lambda \cdot x_{i}=\lambda_{i} x_{i}(1 \leq i \leq n) ; \lambda \cdot y_{i}=\lambda_{i}^{2} y_{i}(1 \leq i \leq n) ;$ and $\lambda \cdot z=\left(\lambda_{1} \cdots \lambda_{n}\right) z$.

There is also an obvious action of the symmetric group $S_{n}$ on $k[\mathbf{x}, \mathbf{y}, z]$ commuting with $\delta_{n}$ : Given $\sigma \in S_{n}$, define

$$
\sigma\left(x_{i}\right)=x_{\sigma(i)} \quad(1 \leq i \leq n) ; \quad \sigma\left(y_{i}\right)=y_{\sigma(i)} \quad(1 \leq i \leq n) ; \quad \text { and } \quad \sigma(z)=z .
$$

Since the actions of the torus $\mathbb{G}_{m}^{n}$ and the symmetric group $S_{n}$ semi-commute with each other, we obtain an action of the group $\left(\mathbb{G}_{m}^{n} \rtimes S_{n}\right)$ on $k[\mathbf{x}, \mathbf{y}, z]$. (In this group, $S_{n}$ acts on $\mathbb{G}_{m}^{n}$ by conjugation.)

The full torus action does not have many invariant functions, and so we consider instead the action of the subgroup $H \subset \mathbb{G}_{m}^{n}$ consisting of elements $\lambda$ such that $\lambda_{1} \cdots \lambda_{n}=1$. Note that $H$ is also a torus, of dimension $(n-1)$. We thus obtain an action of the group $G:=\left(H \rtimes S_{n}\right)$ on $k[\mathbf{x}, \mathbf{y}, z]$.

The ring of invariants $k[\mathbf{x}, \mathbf{y}, z]^{H}$ is generated by monomials in $\mathbf{x}$ and $\mathbf{y}$ whose $\mathbf{y}$-degrees ascend from 0 to $n$, together with $z$. These monomials are:

$$
\begin{aligned}
& \left(x_{1} \cdots x_{n}\right), \\
& x_{i}^{-2}\left(x_{1} \cdots x_{n}\right)^{2} y_{i} \quad(1 \leq i \leq n), \\
& x_{i}^{-2} x_{j}^{-2}\left(x_{1} \cdots x_{n}\right)^{2} y_{i} y_{j} \quad(1 \leq i<j \leq n),
\end{aligned}
$$




$$
\begin{aligned}
& \vdots \\
& \left(x_{i} x_{j}\right)^{2} y_{i}^{-1} y_{j}^{-1}\left(y_{1} \cdots y_{n}\right) \quad(1 \leq i<j \leq n) \\
& x_{i}^{2} y_{i}^{-1}\left(y_{1} \cdots y_{n}\right) \quad(1 \leq i \leq n) \\
& \left(y_{1} \cdots y_{n}\right) .
\end{aligned}
$$

Since $H$ is normal in $G$, the fixed ring of the $G$-action is

$$
k[\mathbf{x}, \mathbf{y}, z]^{G}=\left(k[\mathbf{x}, \mathbf{y}, z]^{H}\right)^{S_{n}}=k\left[s_{0}, s_{1}, \ldots, s_{n}, z\right]=k^{[n+2]},
$$

where $s_{i}$ denotes the sum of the monomials of $\mathbf{y}$-degree $i$ in the list above. The crucial observation is that $k[\mathbf{x}, \mathbf{y}, z]^{G}$ is a polynomial ring, of dimension $n+2$. Since the action of $G$ commutes with $\delta_{n}$, it follows that $\delta_{n}$ restricts to a locally nilpotent derivation of this invariant ring:

$$
\begin{aligned}
& \delta_{n}\left(s_{0}\right)=0, \\
& \delta_{n}\left(s_{1}\right)=n s_{0}^{2}, \\
& \delta_{n}\left(s_{2}\right)=\left(\begin{array}{c}
n \\
2
\end{array}\right) s_{1}, \\
& \vdots \\
& \delta_{n}\left(s_{i}\right)=\left(\begin{array}{c}
n \\
i
\end{array}\right) s_{i-1}, \\
& \vdots \\
& \delta_{n}\left(s_{n}\right)=s_{n-1}, \\
& \delta_{n}(z)=s_{0} .
\end{aligned}
$$

Let $\mathbf{s}=\left(s_{0}, s_{1}, \ldots, s_{n}\right)$, and let $\Delta_{n}$ denote the restriction of $\delta_{n}$ to $k[\mathbf{s}, z]$. Up to scalar multiples, the induced $\mathbb{G}_{a}$-action $\exp \left(t \Delta_{n}\right)$ on $\mathbb{A}^{n+2}=\operatorname{Spec}(k[\mathbf{s}, z])$ is precisely the $\mathbb{G}_{a}$-action on $X=\mathbb{A}^{N+1}$ described in the theorem, where $N=n+1$. It remains to show that the ring of constants $k[\mathbf{s}, z]^{\Delta_{n}}$ is not finitely generated.

In order to do this, we will use the Non-Finiteness Criterion of Daigle and Freudenburg (Lemma 2.1 of [2]; Lemma 7.4 of [5]). This result specifies two conditions that when satisfied, imply that the kernel of a locally nilpotent derivation is not finitely generated.

According to Lemma 2.2 of Kuroda [10, there exists a sequence $\alpha_{i} \in k[\mathbf{x}, \mathbf{y}, z]^{\delta_{n}}$ such that, for some positive integer $e, \alpha_{i}$ has the form $\alpha_{i}=x_{1}^{e} z^{i}+$ (terms of lower degree in $z$ ) for each $i \geq 1$. We may assume each $\alpha_{i}$ is homogeneous (i.e., semi-invariant) relative to the given torus action; otherwise replace $\alpha_{i}$ by the homogeneous summand of $\alpha_{i}$ containing $x_{1}^{e} z^{i}$, which is also in the kernel of $\delta_{n}$. Given $i \geq 1$, let $\beta_{i}$ denote the product of all elements in the orbit of $\alpha_{i}$ under the action of $S_{n}$. Then $\beta_{i} \in k[\mathbf{s}, z]^{\Delta_{n}}$ for each $i$, and has leading $z$-term of the form

$$
\left(\left(x_{1}^{e} z^{i}\right)\left(x_{2}^{e} z^{i}\right) \cdots\left(x_{n}^{e} z^{i}\right)\right)^{(n-1) !}=s_{0}^{e(n-1) !} z^{i n !} .
$$

Therefore, the set of coefficient degrees $\left\{\operatorname{deg} s_{0}^{e(n-1) !}\right\}$ is bounded, while the set $\left\{\operatorname{deg}_{z} z^{i n !}\right\}_{i \geq 1}$ is unbounded. This is the first condition for non-finite generation.

The second condition is that $\delta_{n}(z)=s_{0}$ does not belong to the image of the restriction of $\Delta_{n}$ to the subring $k[\mathbf{s}]$. But this is obvious, since $s_{0}$ does not belong to the ideal $\left(\delta_{n}(\mathbf{s})\right)=\left(s_{0}^{2}, s_{1}, \ldots, s_{n-1}\right)$. By the Non-Finiteness Criterion, it follows that the ring of constants $k[\mathbf{s}, z]^{\Delta_{n}}$ is not finitely generated. 


\section{A Linear COUNTEREXAMPLE IN DIMENSION ELEVEN}

In this section, let $A=k[x, s, t, u, v, z]=k^{[6]}$, and define the triangular derivation $d$ on $A$ by

$$
d x=0, d s=x^{2}, d t=s, d u=t, d v=u, d z=x .
$$

By the results of the preceding section, $A^{d}$ is not finitely generated.

Let $B=k\left[w, x, s_{1}, s_{2}, t_{1}, t_{2}, u_{1}, u_{2}, v_{1}, v_{2}, z\right]=k^{[11]}$, and define commuting linear triangular derivations $\Delta_{1}, \Delta_{2}, \Delta_{3}, \Delta_{4}$ on $B$ by

$$
\begin{aligned}
& \Delta_{1}=\left(x \frac{\partial}{\partial s_{2}}-w \frac{\partial}{\partial s_{1}}\right), \Delta_{2}=\left(x \frac{\partial}{\partial t_{2}}-w \frac{\partial}{\partial t_{1}}\right) \\
& \Delta_{3}=\left(x \frac{\partial}{\partial u_{2}}-w \frac{\partial}{\partial u_{1}}\right), \Delta_{4}=\left(x \frac{\partial}{\partial v_{2}}-w \frac{\partial}{\partial v_{1}}\right) .
\end{aligned}
$$

Their common ring of constants is

$$
\left(\left(\left(B^{\Delta_{4}}\right)^{\Delta_{3}}\right)^{\Delta_{2}}\right)^{\Delta_{1}}=k\left[w, x, x s_{1}+w s_{2}, x t_{1}+w t_{2}, x u_{1}+w u_{2}, x v_{1}+w v_{2}, z\right] \cong k^{[7]} \text {. }
$$

This is due to the simple fact that, for any base $\operatorname{ring} R$, if $\theta$ is the $R$-derivation of $R[X, Y]=R^{[2]}$ defined by $\theta X=a$ and $\theta Y=-b$ for some $a, b \in R$ (not both 0 ), then $R[X, Y]^{\theta}=R[b X+a Y] \cong R^{[1]}$. Applying this four times in succession gives the equality above.

In addition, define a fifth linear triangular derivation $\mathcal{D}$ of $B$ by

$$
\mathcal{D}=x \frac{\partial}{\partial s_{1}}+s_{1} \frac{\partial}{\partial t_{1}}+t_{1} \frac{\partial}{\partial u_{1}}+u_{1} \frac{\partial}{\partial v_{1}}+s_{2} \frac{\partial}{\partial t_{2}}+t_{2} \frac{\partial}{\partial u_{2}}+u_{2} \frac{\partial}{\partial v_{2}}+x \frac{\partial}{\partial z} .
$$

In the Lie algebra of $k$-derivations of $B$, we have relations

$$
\left[\Delta_{1}, \mathcal{D}\right]=\Delta_{2}, \quad\left[\Delta_{2}, \mathcal{D}\right]=\Delta_{3}, \quad\left[\Delta_{3}, \mathcal{D}\right]=\Delta_{4}, \quad\left[\Delta_{4}, \mathcal{D}\right]=0
$$

So these 5 linear derivations form a Lie subalgebra.

In particular, let $\mathfrak{h}$ denote the subalgebra

$$
\mathfrak{h}=\left(k \Delta_{1} \oplus k \Delta_{2} \oplus k \Delta_{3} \oplus k \Delta_{4}\right)
$$

and let $\mathfrak{g}=\mathfrak{h} \oplus k \mathcal{D}$. Then the group $\Gamma:=\exp \mathfrak{g}$ acts linearly on $\mathbb{A}^{11}$. Let $\Omega$ denote the subgroup $\Omega=\exp \mathfrak{h}$, which is isomorphic to the vector group $\mathbb{G}_{a}^{4}$.

Note that the equations (11) above show that the adjoint $[\cdot, \mathcal{D}]$ defines the basic linear derivation of $\mathfrak{h}$. It follows that $\Omega$ is normal in $\Gamma$; the subgroup $\exp t[\cdot, \mathcal{D}] \subset \Gamma$ acts on $\Omega$; and $\Gamma \cong\left(\mathbb{G}_{a}^{4} \rtimes \mathbb{G}_{a}\right)$.

This means that $\mathcal{D}$ restricts to the subring $B^{\Omega}=k^{[7]}$, and is given by

$$
\begin{aligned}
& \mathcal{D} w=0, \\
& \mathcal{D} x=0, \\
& \mathcal{D}\left(x s_{1}+w s_{2}\right)=x^{2}, \\
& \mathcal{D}\left(x t_{1}+w t_{2}\right)=x s_{1}+w s_{2}, \\
& \mathcal{D}\left(x u_{1}+w u_{2}\right)=x t_{1}+w t_{2}, \\
& \mathcal{D}\left(x v_{1}+w v_{2}\right)=x u_{1}+w u_{2}, \\
& \mathcal{D} z=x .
\end{aligned}
$$

This is manifestly the same as the derivation $d$, defined at the beginning of this section, extended to the ring $A[w]=A^{[1]}$ by $d w=0$. Therefore,

$$
B^{\Gamma}=\left(B^{\Omega}\right)^{\mathcal{D}} \cong(A[w])^{d}=\left(A^{d}\right)[w] \cong\left(A^{d}\right)^{[1]},
$$

and this ring is not finitely generated over $k$. We have thus proved the following. 
Theorem 4.1. Let $B=k^{[11]}$, and let $\mathfrak{g}$ be the Lie algebra generated by the linear derivations $\Delta_{1}, \Delta_{2}, \Delta_{3}, \Delta_{4}$ and $\mathcal{D}$ of $B$, as defined above. Then the group $\Gamma=$ $\exp \mathfrak{g} \cong\left(\mathbb{G}_{a}^{4} \rtimes \mathbb{G}_{a}\right)$ acts algebraically on $V=\mathbb{A}^{11}$ by linear transformations, and its invariant ring $k[V]^{\Gamma}$ is not finitely generated.

Remark. Using Theorem 3.1, the same reasoning yields, for each integer $n \geq 4$, a linear action of the group $\Gamma_{n}=\left(\mathbb{G}_{a}^{n} \rtimes \mathbb{G}_{a}\right)$ on $V_{n}=\mathbb{A}^{2 n+3}$ such that $k\left[V_{n}\right]^{\Gamma_{n}}$ is not finitely generated.

\section{REFERENCES}

1. A. A'Campo-Neuen, Note on a counterexample to Hilbert's fourteenth problem given by P. Roberts, Indag. Math., N.S. 5 (1994), 253-257. MR.1298772 (95k:13003)

2. D. Daigle and G. Freudenburg, A counterexample to Hilbert's Fourteenth Problem in dimension five, J. Algebra 221 (1999), 528-535. MR.1728394 (2000i:13029)

3. H. Derksen and G. Kemper, Computational Invariant Theory, Springer Verlag, Berlin, Heidelberg, New York, 2002. MR.1918599 (2003g:13004)

4. A. van den Essen, Polynomial Automorphisms and the Jacobian Conjecture, Birkhauser, Boston, 2000. MR 1790619 (2001j:14082)

5. G. Freudenburg, Locally Nilpotent Derivations and $\mathbb{G}_{a}$-Actions, Springer-Verlag, New York, (to appear).

6. J. Khoury, On some properties of elementary derivations in dimension six, J. Pure Appl. Algebra 156 (2001), 69-79. MR1807016 (2001m:13045)

7. H. Kojima and M. Miyanishi, On P. Roberts' counterexample to the fourteenth problem of Hilbert, J. Pure Appl. Algebra 122 (1997), 247-268. MR1481092 (98j:13008)

8. K. Kurano, Positive characteristic finite generatiion of symbolic Rees algebra and Roberts' counterexamples to the fourteenth problem of Hilbert, Tokyo J. Math. 16 (1993), 473-496. MR.1247667 (94k:13004)

9. S. Kuroda, A counterexample to the Fourteenth Problem of Hilbert in dimension four, J. Algebra 279 (2004), 126-134. MR2078390 (2005f:13022)

10. _ A generalization of Roberts' counterexample to the fourteenth problem of Hilbert, Tohoku Math. J. 56 (2004), 501-522. MR2097158 (2005i:13008)

11. _ A counterexample to the Fourteenth Problem of Hilbert in dimension three, Michigan Math. J. 53 (2005), 123-132. MR2125538 (2005k:13043)

12. S. Maubach, Triangular monomial derivations on $k\left[x_{1}, x_{2}, x_{3}, x_{4}\right]$ have kernel generated by at most four elements, J. Pure Appl. Algebra 153 (2000), 165-170. MR.1780741 (2001g:13057)

13. S. Mukai, Geometric realization of T-shaped root systems and counterexamples to Hilbert's fourteenth problem, Algebraic Transformation Groups and Algebraic Varieties, SpringerVerlag, Berlin, 2004, Encyclopaedia Math. Sci. 132, 123-129. MR2090672 (2005h:13008)

14. M. Nagata, On the Fourteenth Problem of Hilbert, Proc. I.C.M. 1958, Cambridge University Press, 1960, pp. 459-462. MR0116056 (22:6851)

15. P. Roberts, An infinitely generated symbolic blow-up in a power series ring and a new counterexample to Hilbert's fourteenth problem, J. Algebra 132 (1990), 461-473. MR1061491 (91j:13006)

16. R. Steinberg, Nagata's example, Algebraic Groups and Lie Groups, Cambridge University Press, 1997, pp. 375-384.

17. R. Tanimoto, Linear counterexamples to the fourteenth problem of Hilbert, J. Algebra 275 (2004), 331-338. MR2047451 (2005g:13012)

18. O. Zariski, Interpretations algebrico-geometriques du quatorzieme problem de Hilbert, Bull. Sci. Math. 78 (1954), 155-168. MR0065217 (16:398c)

Department of Mathematics, Western Michigan University, Kalamazoo, Michigan 49008

E-mail address: gene.freudenburg@umich.edu 